\title{
Minimally Invasive Hemithyroidectomy Using a Mini Incision over the Upper Pole of Thyroid Swelling
}

\author{
M. Subrahmanyam*, R. Sirisha, A. Deepthi, R. N. Mishra \\ Department of General surgery, Kamineni Institute of Medical Sciences, Narketpally, T.S, India
}

Email address:

subrahmanyam_mutya@yahoomail.com (M. Subrahmanyam)

To cite this article:

M. Subrahmanyam, R. Sirisha, A. Deepthi, R. N. Mishra. Minimally Invasive Hemithyroidectomy Using a Mini Incision over the Upper Pole of Thyroid Swelling. Journal of Surgery. Vol. 3, No. 3, 2015, pp. 21-25. doi: 10.11648/j.js.20150303.12

\begin{abstract}
Minimally invasive thyroid surgery, using various techniques including endoscopic and video-assisted have been reported. Thyroid surgery using a mini-incision over the upper pole of the thyroid, as a new technique is presented here. Methods: The study group comprised of 52 patients undergoing minimally invasive thyroid surgery (MITS) by open method during the period May 2005-May 2013. Data regarding patient demographics, indication for surgery, operation performed, nodule size, final pathology, and complications were recorded. The operation was carried out through a $1.5-2-\mathrm{cm}$ incision placed directly over the upper pole of the swelling, and deepening the incision to visualize the superior pedicle. After ligating the superior pedicle, the finger is passed over the gland and separated from all sides. Then the thyroid is pulled up and the inferior pedicle accessed and ligated. Any bleeding points were taken care of and the wound was closed without drain. Results: Fifty two patients underwent MITS, 38 women and fourteen men. All the patients underwent hemi -thyroidectomy. The average measured incision size was $2 . \mathrm{cm}$ at the end of the procedure. The average nodule size was $3.2 \mathrm{~cm}$, and the average thyroid lobe resected measured $4.5 \mathrm{~cm}$ in maximal length. Final pathology revealed follicular adenoma in 51 patients and one thyroid cancer (follicular). There was one wound infection and one patient had temporary recurrent laryngeal nerve neurapraxia. Conclusion: Minimally invasive thyroid surgery with a minimal incision over the upper pole of thyroid swelling as an alternative to open thyroid surgery, using a standard cervical collar incision, is safe and feasible.
\end{abstract}

Keywords: Minimally Invasive Surgery, Hemithyroidectomy, Follicular Adenoma, Minimal Access Thyroid Surgery

\section{Introduction}

Thyroid surgery is the most common procedure done in the neck. Kocher in 1909, pioneered what is today known as the conventional thyroidectomy [1]. The goal for the surgeon is to remove the whole gland or its specific part, preserving inferior and superior laryngeal nerves and parathyroid glands, while achieving safe hemostasis mainly by ligating superior and inferior thyroid arteries[2]. Since Gagner et al described endoscopic approach to the parathyroid glands, various techniques have been described and popularized for thyroid surgery[3].Minimally invasive video assisted thyroidectomy(MIVAT)was introduced by Miccoli et al $[4,5]$ who reported significant reduction in postoperative pain and better cosmetic results. Minimally invasive nonendoscopic thyroidectomy (MINET, MIT)is also known as small incision thyroidetomy and do not require specialized instruments like endoscopes and videoassistance. Ferzli et al first reported MINET with $2.5 \mathrm{~cm}$. cervical incision, using head light for visualization[6]. Reduced tissue trauma, shorter hospital stay, better cosmetic results, minimal postoperative pain and patients comfort are the advantages with MITS[7].

Patient selection is the most important step for the success of any MITS technique for both benign and malignant thyroid swellings. For MIVAT, the criteria are thyroid nodules less than $30 \mathrm{~mm}$ in largest diameter, thyroid gland volume less than $25 \mathrm{ml}$, absence of thyroid gland fixation, low risk papillary carcinoma, no previous irradiation or surgery[4]. Though there are no special criteria for MINET, volume of the gland and extent of its fixation to the surrounding structures are important factors to be considered. Minimal invasive thyroid surgery is usually done through a direct lateral mini-incision approach or a central mini- incision approach[1]. We report our experience using a mini-incision over the upper pole of 
thyroid swelling .and assessed the results.

\section{Patients and Methods}

A group of 52 patients with thyroid nodule treated at the General Hospital, Sangli and Kamineni Institute of Medical sciences Hospital from May 2005 to May 2013 formed material of this study. Data regarding patient demographics, indication for surgery, operation performed, nodule size, final pathology, and complications were recorded. Exclusion criteria for this procedure included: family history of thyroid cancer, previous neck irradiation or surgery, carcinoma on fine needle aspiration, presence of significant thyroiditis, multi-nodular goiter, and nodule size $>3 \mathrm{~cm}$. Institute ethical committee have approved this study and informed consent has been taken from the patients. Thyroid function tests, FNAC, X-ray neck, ultra sound of the neck and indirect laryngoscopy, to see the movements of the vocal cords were performed.

Hemi - thyroidectomy was carried out under general anaesthesia. The neck was extended. A $1.5-2 \mathrm{~cm}$ transverse incision was placed directly over the upper pole of the swelling. The platysma was divided. The deep fascia and the strap muscles of the neck were divided. With gentle dissection the upper pole was identified. The superior pedicle was ligated avoiding the external laryngeal nerve and divided. The upper pole of the thyroid was pulled up gently and a space was created over the surface of the gland by introducing the finger downwards towards the lower pole. Then the finger is rotated towards either side and separated. When once this is achieved, the lobe of the thyroid is lifted with the help of a Babcock forceps or artery forceps. By slipping the finger behind the thyroid lobe and by gentle dissection, it was separated posteriorly down up to the lower pole of the gland. After the whole lobe is lifted, it was gently pulled up and the inferior pedicle was identified, ligated and divided by avoiding the recurrent laryngeal nerve. The lobe along with the isthmus was removed by gently pulling up and brought out through the incision. The bleeding points if any were taken care of and the wound was closed without a drain. A thyroid bandage was applied and the patient was observed for any complications. Fig 1-4 shows the steps of the operation. Pain was assessed by visual analogue scale from $0-10$ (VAS0-10,0 corresponding to no pain, 10 corresponding to the most severe pain, which patient is able to imagine.Pain was measured at the end of 1 st, 6th, 12th post-operative hour. Indirect laryngosopy was done post-operatively to evaluate inferior laryngeal nerve function. The patients were followed up to see the cosmetic result and any other complaint.

\section{Results}

There were 38 women and fourteen men with an age range of 25-62 years (mean 38.2).All the patients, on clinical examination were found to be having solitary thyroid nodule with euthyroid status. FNAC was follicular adenoma in all patients. Ultra sonography revealed solid lesions in all the cases. Thyroid functions were within normal limits confirming the clinical examination. X-ray neck did not show deviation of trachea or calcification in any case. Indirect laryngoscopy revealed vocal cord movements to be normal.

Hemithyrodectomy was easily done by this procedure. No difficulty was encountered in getting the plane over the front of thyroid lobe as well as behind. The incision in all the operations directly exposed the superior thyroid artery with the vein, which could be liagated easily.(Fig.1). The blood loss was minimal average $8.5 \mathrm{ml}$.(range $5-10 \mathrm{ml}$ ). The incision size measured at the end of the operation was 1.64 $\mathrm{cm}$, (range 1.5-2 $\mathrm{cm}$ ) . The average nodule size was $3.2 \mathrm{~cm}$ (range $2.5-4 \mathrm{~cm}$ ) and the average thyroid lobe resected measured $4.5 \mathrm{~cm}$ in maximal length. Final pathology revealed follicular adenoma in 51 patients and one thyroid cancer (follicular).

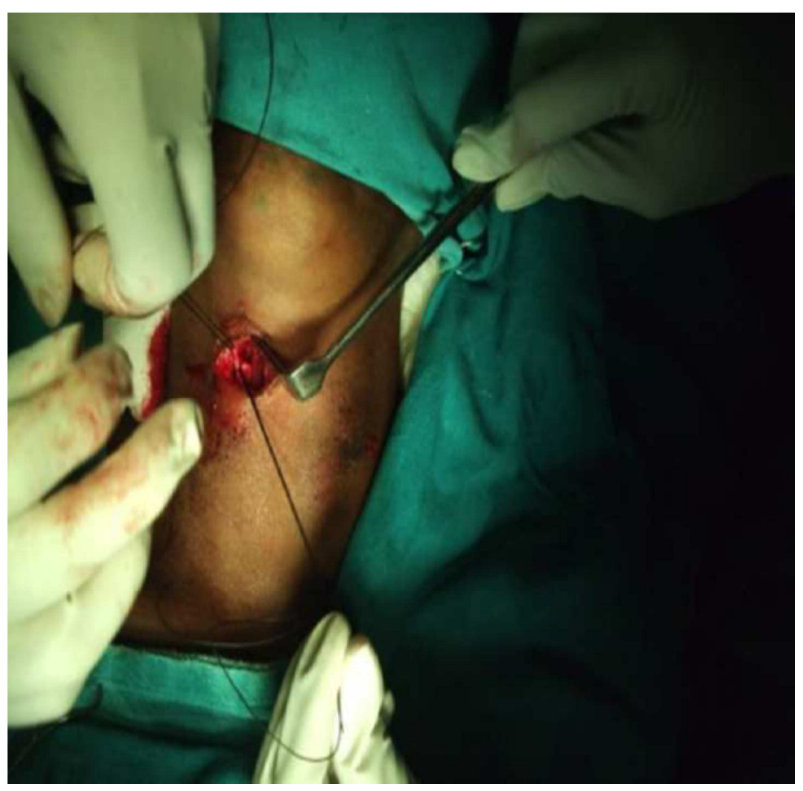

Fig. 1. shows ligation of superior thyroid artery.

There was no difficulty in identification of superior pedicle and it could be easily ligated. There was no bleeding while separating the gland from all sides. The inferior thyroid artery and the vein could be easily ligated by pulling up, there was no tearing of vessels or bleeding from other causes. ( Figs .2-4). At the end of the operation, after removing the endotracheal tube,in one patient, there was restricted movement of the vocal cord on that side due to recurrent laryngeal nerve injury due to neurapraxia which recovered after 2 -weeks. The mean operation time was 38 minutes (range 25-40 minutes)

There was no postoperative hematoma in any of the patients, and there was no soakage of the wound.

The patients had no significant pain, (ranging from $0-1$ as per visual analogue scale).

32 patients could be followed up to 4-6 years, 12 from 2-4 years and 6from 6 months to 6 years, and 2 patients lost for 
follow up. Of the patients that were followed up, the cosmetic result was excellent as assessed by the patients themselves except in one patient who had wound infection One patient who had carcinoma underwent total thyroidectomy. On follow up, there was no recurrence of thyroid swelling.

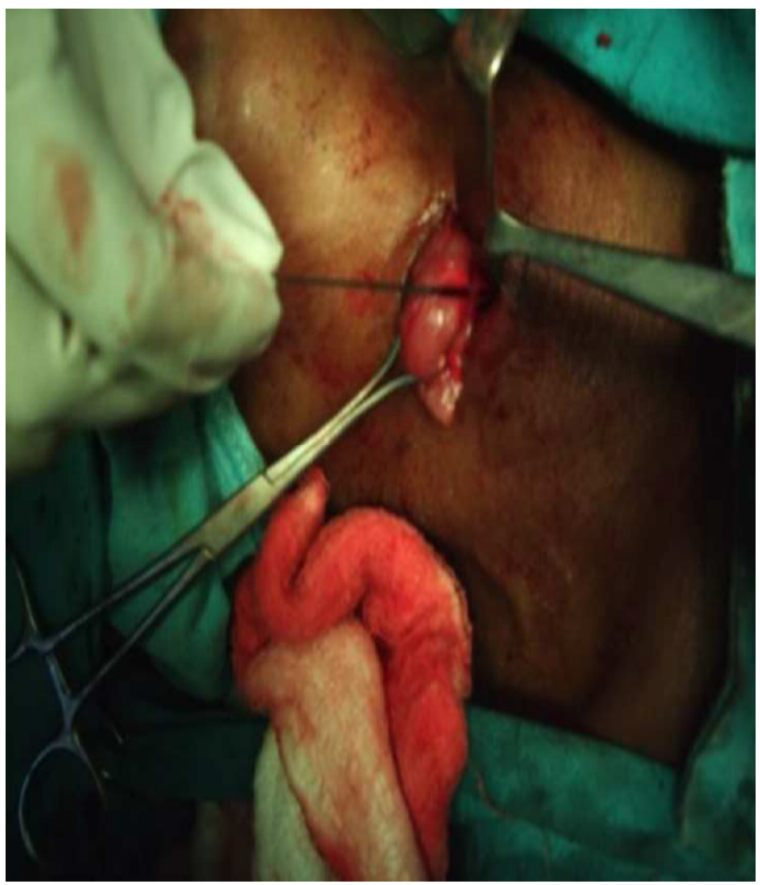

Fig. 2. shows mobilisation of thyroid.

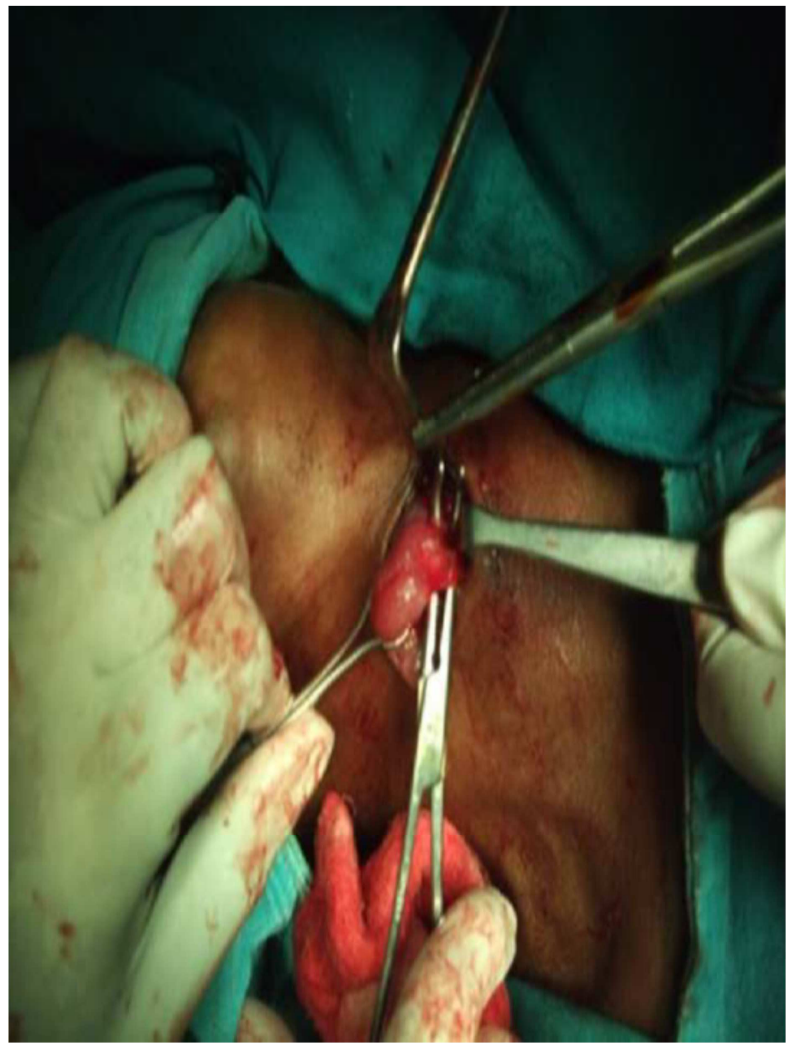

Fig. 3. shows ligation of inferior thyroid artery.

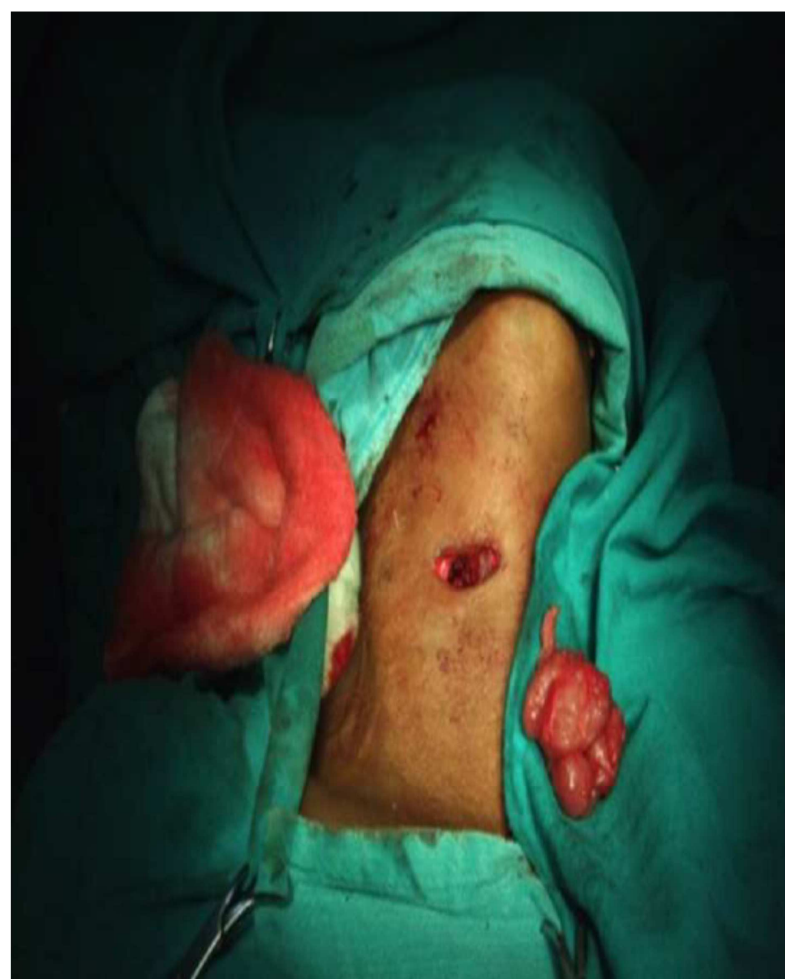

Fig. 4. shows incision after hemithyroidectomy.

\section{Discussion}

Thyroid surgery has evolved considerably from the times of Billroth and Kocher due to better understanding of the surgical principles, better equipment and advanced surgical techniques. Kocher, in 1909, pioneered what is today known as the conventional thyroidectomy [1]. It has remained the standard approach to the thyroid gland and is still the most widely used technique world-wide. A recent advance is minimal access thyroid surgery (MATS). The concept of minimal access surgery is not new and it has been practiced in many other surgical specialties for over two decades now. It is attractive because patients are concerned not only about the results of treating their thyroid disease, but also outcomes such as better cosmesis, reduced hospital stay and decreased pain.

The three most commonly utilized approaches for thyroid surgery are minimally invasive video-assisted thyroidectomy (MIVAT), minimal invasive thyroid surgery through a direct lateral mini-incision approach (MITS) or a central miniincision approach. The major application of this surgical approach is the diagnostic excision of solitary thyroid nodules with atypical follicular cytology or excision of toxic thyroid nodules. In relation to the diagnostic excision of atypical solitary nodules, MITS should be seen as an investigative procedure positioned between fine-needle aspiration biopsy (FNAB) and conventional standard thyroidectomy achieved through a 5-6-cm incision. MITS follows the same principles as a conventional thyroid surgical procedure but is performed with less trauma of access. It uses a capsular dissection and requires identification of the 
laryngeal nerves and parathyroid glands. The simple MITS operative setup combined with its low costs has made it an attractive alternative treatment option to conventional thyroidectomy.[8-10].In the present study, an incision was made over the upper pole of the thyroid swelling and it was possible to directly reach the superior thyroid artery and ligate it. It was easy to perform and time consumed was less and with less complications.

The term minimally invasive thyroidectomy for open surgeries is used only to describe thyroid and parathyroid procedures that are routinely associated with an incision shorter than $3.0 \mathrm{~cm}$ for thyroidectomy and $2.5 \mathrm{~cm}$ for parathyroidectomy[11]. Various thyroidectomy approaches via the neck, axilla, chest wall, and breast have been reported. These new techniques are compared with the fact that they have to compete with conventional thyroidectomy, with its high standard of treatment in terms of both efficacy and safety of performance. Among the cervical approaches the minimally invasive video-assisted thyroidectomy technique is the method that has so far become most widespread and is considered the most easily consistent procedure of its class of operations. Limiting factors of all cervical approaches include the length of the incision and consequently the size of the specimen resected. Furthermore, rather than the length of the neck incision, the quality of the scar, including its potential drawbacks, is a determinant for the cosmetic result.[11-15]

Technique of conventional thyroidectomy is being performed today in the same way as described at that time by Billroth, Kocher and Halsted[1].

The introduction of new technologies during the late 20th century, such as intra operative neuro-monitoring or harmonic scalpel can be considered as innovative aspects that improved safety in thyroid resection with mortality rates approaching zero and morbidity in high volume units being $<1 \%$. The advent of minimally invasive surgery and its application in thyroid surgery represents a truly qualitative great leap forward aiming to reduce pain and improve cosmetics. Various thyroidectomy approaches via the neck, axilla, chest wall, and breast have been reported. These new techniques are confronted with the fact that they have to compete with conventional thyroidectomy with its high standard of treatment on terms of both efficacy and safety[16].

The fact that many different endoscopic techniques have been described and that none seem to be universally accepted may be a direct result of technical difficulties and unproven superiority of one technique compared with another. Currently, it is not possible to make an evidence-based recommendation since superiority of endoscopic to conventional thyroidectomy has not yet been demonstrated. Limiting factors of the MIVAT technique with its bothersome $20 \mathrm{~mm}$ cervical incision and the specimen size to remove on one side and the maximally invasive character of the extracervical approaches on the other side made us to think about a method that should fulfill the general criteria.

By the technique described here, we are able to reduce operation time, no drain was required in any patient and with good cosmesis. There was only one recurrent laryngeal nerve neurapraxia which recovered later. By proper selection of the patient, minimally invasive surgery through a small incision over the upper part of the selling is feasible, safe and easy to perform with a small learning curve.

\section{Conclusion}

Hemi thyroidectomy with a mini incision over the upper part of the swelling as an alternative to open thyroid surgery, using a standard cervical collar incision, is safe and feasible.

\section{References}

[1] Kazi R,Katna R,Dwivedi RC, Minimal access thyroid surgerya new dawn? Ann R Coll Surg Engl 2010;92:361-2.

[2] Fik Z,Astl J ,Zabrodsky M, et al. Minimally invasive videoassisted versus minimally invasive non endoscopic thyroidectomy,Biomed Research Inaternational2014, Article ID 450170, 7 pages, 2014. doi:10.1155/2014/450170

[3] Gagner M. Endoscopic subtotal parathyroidectomy in patients with primary hyperparathyroidism, British Journal of Surgery, 1996,83:875.

[4] Miccoli P, Berti P, Conte,M et al., Minimally invasive surgery for thyroid small nodules: preliminary report ,Journal of Endocrinological Investigation, 1999,22:849-851.

[5] MiccoliP, Berti P, . Frustaci GL et al. Video-assisted thyroidectomy: indications and results.Langenbeck's Archieves of Surgery, 2006,39:168-71.

[6] Ferzli GS, Sayad P . Abdo Z et al. Minimally invasive, nonendoscopic thyroid surgery. Journal of the American College of Surgeons.2001,192:665-8.

[7] Norman J ,Chheda H, Farrell C. Minimally invasive parathyroidectomy for primary hyperparathyroidism: decreasing operation time and potential complications while improving cosmetic result., The Americ Surgeon, 1998,64: 391-96

[8] Touzopoulos P, Karanikas M, ZarogoulidisP, et al. Current surgical status of thyroid diseases, Journal of Multidisciplinary Health care,2011, 4:441-49.

[9] Ikeda Y, Takami,H ,NiimiM,et al...Endoscopic thyroidectomy by the axillary approach, Surgical Endoscopy, 2001: 15, 1362-64.

[10] Karakas T. Steinfeldt, .Gockel A et al. Transoral thyroid and parathyroid surgery-development of a new transoral technique, Surgery, 2011:150, 108-15.

[11] Brunaud L, Zarnegar R, Wada.N, et al. Incision length for standard thyroidectomy and parathyroidectomy: when is it minimally invasive? Archives of Surgery, 2003; 138:1140-43.

[12] Miccoli.P, Pinchera.A, Materazzi.G et al., Surgical treatment of low- and i ntermediate-risk papillary thyroid cancer with minimally invasive video-assisted thyroidectomy,.The Journal of Clinical Endocrinology \& Metabolism,2009; 94, 1618-22 . 
[13] Miccoli P, Minuto M.N, Berti.P.et al. Update on the diagnosis and treatment of differentiated thyroid cance., Quarterly Journal of Nuclear Medicine and Molecular Imaging, 2009; 53. 465-72.

[14] Zabrodsky M, BoucekJ, KastnerJ. et al. Immediate revision in patients with bilateral recurrent laryngeal nerve palsy after thyroid and parathyroid surgery. How worthy is it? Acta Otorhinolaryngologica Italica, 2012: 32 222-28.
[15] Alvarado R, McMullenT, Sidhu SB et al. Minimally invasive thyroid surgery for single nodules: an evidence-based review of the lateral mini-incision technique, World Journal of Surgery, 2008:32, 1341-48.

[16] Dhiman SV, Inabnet W., Minimally invasive surgery for thyroid diseases and thyroid cancer, Journal of Surgical Oncology, 2008: 97 665-68. 